\title{
Importance of experimental information (metadata) for archived sequence data: case of specific gene bias due to lag time between sample harvest and RNA protection in RNA sequencing
}

\author{
Tomoko Matsuda ${ }^{\text {Corresp. } 1}$ \\ ${ }^{1}$ Nihon BioData Corporation, Kawasaki, Kanagawa, Japan \\ Corresponding Author: Tomoko Matsuda \\ Email address: tomokom@nbiodata.com
}

Large volumes of high-throughput sequencing data have been submitted to the Sequencing Read Archive (SRA). The lack of experimental metadata associated with the data makes reuse and understanding data quality very difficult. In the case of RNA sequencing (RNA-Seq), which reveals the presence and quantity of RNA in a biological sample at any moment, it is necessary to consider that gene expression responds over a short time interval (several seconds to a few minutes) in many organisms. Therefore, to isolate RNA that accurately reflects the transcriptome at the point of harvest, raw biological samples should be processed by freezing in liquid nitrogen, immersing in RNA stabilization reagent or lysing and homogenizing in RNA lysis buffer containing guanidine thiocyanate as soon as possible. As the number of samples handled simultaneously increases, the time until the RNA is protected can increase. Here, to evaluate the effect of different lag times in RNA protection on RNA-Seq data, we harvested CHO-S cells after 3, 5, 6 , and 7 days of cultivation, added RNA lysis buffer in a time course of $15,30,45$, and 60 min after harvest, and conducted RNA-Seq. These RNA samples showed high RNA integrity number (RIN) values indicating non-degraded RNA, and sequence data from libraries prepared with these RNA samples was of high quality according to FastQC. We observed that, at the same cultivation day, global trends of gene expression were similar across the time course of addition of RNA lysis buffer; however, the expression of some genes was significantly different between the time-course samples of the same cultivation day; most of these differentially expressed genes were related to apoptosis. We conclude that the time lag between sample harvest and RNA protection influences gene expression of specific genes. It is, therefore, necessary to know not only RIN values of RNA and the quality of the sequence data but also how the experiment was performed when acquiring RNA-Seq data from the database. 
2 Importance of experimental information (metadata) for

3 archived sequence data: case of specific gene bias

4 due to lag time between sample harvest and RNA

5 protection in RNA sequencing

6

7

8

9

10

11

12

13

14

15

16

\section{7}

18

19

20

21

22

23

24

25

26

27

28

29

30

31

32

33

34

35

36

37

Tomoko Matsuda

Nihon BioData Corporation, Kawasaki, Kanagawa, Japan

Corresponding Author:

Tomoko Matsuda ${ }^{1}$

Tech-Pot 4-2 KSP West 2F 3-2-1 Sakado Takatsu-ku, Kawasaki Kanagawa, 213-0012, Japan

Email address: tomokom@nbiodata.com

\section{Abstract}

Large volumes of high-throughput sequencing data have been submitted to the Sequencing Read Archive (SRA). The lack of experimental metadata associated with the data makes reuse and understanding data quality very difficult. In the case of RNA sequencing (RNA-Seq), which reveals the presence and quantity of RNA in a biological sample at any moment, it is necessary to consider that gene expression responds over a short time interval (several seconds to a few minutes) in many organisms. Therefore, to isolate RNA that accurately reflects the transcriptome at the point of harvest, raw biological samples should be processed by freezing in liquid nitrogen, immersing in RNA stabilization reagent or lysing and homogenizing in RNA lysis buffer containing guanidine thiocyanate as soon as possible. As the number of samples handled simultaneously increases, the time until the RNA is protected can increase. Here, to evaluate the effect of different lag times in RNA protection on RNA-Seq data, we harvested CHO-S cells after $3,5,6$, and 7 days of cultivation, added RNA lysis buffer in a time course of 15,30,45, and 60 min after harvest, and conducted RNA-Seq. These RNA samples showed high RNA integrity number (RIN) values indicating non-degraded RNA, and sequence data from libraries prepared with these RNA samples was of high quality according to FastQC. We observed that, at the same cultivation day, global trends of gene expression were similar across the time course of addition of RNA lysis buffer; however, the expression of some genes was significantly different between the time-course samples of the same cultivation day; most of these differentially expressed genes were related to apoptosis. We conclude that the time lag between sample harvest and RNA protection influences gene expression of specific genes. It is, therefore, necessary to know not 
only RIN values of RNA and the quality of the sequence data but also how the experiment was performed when acquiring RNA-Seq data from the database.

\section{Introduction}

High-throughput sequencing (HTS) has been applied in several fields of biological research since its appearance about a decade ago and has made it possible to collect data with substantially higher-throughput and lower-cost (Kircher \& Kelso, 2010; Kodama et al., 2012). Large volumes of HTS data have been submitted to the Sequence Read Archive (SRA, Leinonen, Sugawara \& Shumway, 2010), and repository users can access the archived data for reuse. Nakazato, Ohta \& Bono (2013) constructed a publication list including PubMed IDs (PMIDs) referring to SRA entries. This list is useful for retrieving archived data based on the experimental details (metadata); however, metadata described by the data depositor lack some important information about protocol steps (Alnasir \& Shanahan, 2015). Ohta, Nakazato \& Bono (2017) calculated the quality of all archived sequence data in the SRA to allow users to control not only metadata but also the data quality in their searches.

It may not be possible to clarify the validity of the sequence data by examining the quality control data generated by a quality control tool (e.g., FastQC) without metadata containing experiment-specific information. For example, this author has obtained two RNA samples one isolated from cultured cells in $30 \mathrm{~min}$ after sampling, the other isolated from same cells in 60 min after sampling; both of these RNA samples prepared in the same laboratory showed high RNA integrity number (RIN) values indicating non-degraded RNA. However, it remains questionable whether data obtained from RNA samples with different time lags from sample harvest to RNA protection can be handled equally. In the case of RNA sequencing (RNA-Seq), it is necessary to consider that changes in gene expression can occur over a short time period (several seconds to a few minutes) in many organisms. For instance, in the filamentous fungus Aspergillus fumigatus, $23 \%$ and $35 \%$ of genes were differentially expressed after 30 and 120 min of hypoxia exposure, respectively (Losada et al., 2014) compared with before exposure. In the case of cultured cells, dynamic changes of gene expression profiles occurred during the first $2 \mathrm{~h}$ $(30,60,90$, and $120 \mathrm{~min})$ of serum stimulation in hTERT immortalized foreskin-derived human fibroblasts (Kirkconnell et al., 2016). Therefore, to isolate RNA that accurately reflects the transcriptome at the point of harvest, raw biological samples should be frozen in liquid nitrogen or treated with RNA stabilization reagent or RNA lysis buffer as soon as possible.

Some cultured mammalian cells need to be suspended in $1 \times$ PBS to remove fetal bovine serum and/or media before treatment with RNA lysis buffer. For example, the strategy for RNA sample processing from suspended Chinese hamster ovary $(\mathrm{CHO})$ cells at the author's laboratory at Nihon BioData Corporation is as follows: 1) The medium containing the suspended CHO cells is transferred from the cell culture flask to a $1.5 \mathrm{~mL}$ centrifuge tube. 2) The centrifuge tube is 
78

79

80

81

82

83

84

85

86

87

88

89

90

91

92

93

94

95

96

97

98

99

100

101

102

103

104

105

106

107

108

109

110

111

112

113

114

115

116

117

centrifuged for $5 \mathrm{~min}$ at $300 \times \mathrm{g}$ and all supernatant is removed. 3) $200 \mu \mathrm{L}$ of $1 \times$ PBS is added to the cell pellet and pipetted up and down five times to thoroughly suspend the pellet. 4) The centrifuge tube is centrifuged for $5 \mathrm{~min}$ at $300 \times g$ and all supernatant is removed. 5) The centrifuge tube is flicked to loosen the cell pellet from the bottom. 6) $350 \mu \mathrm{L}$ of buffer RLT (RNA lysis buffer containing guanidine thiocyanate; QIAGEN, Hilden, Germany) is added to the pellet and pipetted up and down five times to mix thoroughly. 7) The entire cell suspension was immediately loaded onto a QIAshredder spin column (QIAGEN) and homogenized by centrifugation for $2 \mathrm{~min}$ in a microcentrifuge at maximum speed. When cells are treated by using the above strategy, the time lag until the addition of RNA lysis buffer, which contains guanidine hydrochloride, is critical. Because RNA in cells suspended in RNA lysis buffer immediately inactivates RNases to ensure isolation of intact RNA, the time lag until addition of this reagent should be as short as possible. If the number of samples is less than 10, RNA lysis buffer can be added to the cell pellet within about $15 \mathrm{~min}$. As the number of samples increases, however, the time it takes for centrifuging and pipette operation for samples becomes longer. As a result, the cells remain in media or $1 \times \mathrm{PBS}$ at the conditions (temperature and $\mathrm{CO}_{2}$ concentration) outside the incubator for a long time.

Here, to evaluate whether RNA-Seq data obtained from RNA samples with a different lag time until RNA protection can be analyzed equally, we harvested CHO-S cells after 3, 5, 6, and 7 days of cultivation, added RNA lysis buffer at various lag times (Fig. 1; 15, 30, 45, and $60 \mathrm{~min}$ ), purified the RNA, and then performed RNA-Seq. We found that the global trends of gene expression were similar, but the expression of some specific genes was significantly different between samples that were processed with different lag times until RNA protection on the same cultivation day. Most of the genes whose profiles changed with lag time until RNA protection were related to apoptosis. Based on our results, we propose to describe detailed protocol procedures including working time for reusing sequence data accessed from the data repository.

\section{Materials \& Methods}

Cells

Bach CHO-S (Thermo Fisher Scientific, Waltham, MA, USA) cell culture was grown in $20 \mathrm{ml}$ of Gibco CD-CHO medium (Thermo Fisher Scientific) containing $400 \mu \mathrm{L}$ of $200 \mathrm{mM}$ L-Alanyl-Lglutamine (final concentration: $4 \mathrm{mM}$; Sigma-Aldrich, St. Louis, MI, USA) and $40 \mu \mathrm{L}$ of Gibco Anti-Clumping Agent (final concentration, $0.2 \%(\mathrm{v} / \mathrm{v})$; Thermo Fisher Scientific) in a $125 \mathrm{~mL}$ flask. The flask was shaken $(120 \mathrm{rpm})$ at $37^{\circ} \mathrm{C}$ in a humidified $5 \% \mathrm{CO}_{2}$ atmosphere. Determination of total and viable cell numbers was performed with Vi-Cell XR Cell Viability Analyzer (Beckman Coulter, Fullerton, CA, USA) and CellProfiler software (Carpenter et al., 2006). The images obtained from Vi-Cell XR were analyzed and total and viable cell numbers were counted using the CellProfiler pipeline. The growth curve of CHO-S cells over the period of study is shown in Fig. 2. 

addition of RNA lysis buffer. Two persons performed this experiment: one person opened and closed the lids of the tubes, added reagent, and carried out the pipetting, and another put the tubes in and out of a microcentrifuge. The experiment was conducted as follows (Fig. 1). Three technical replicates for every sample were prepared. The medium containing the suspended $\mathrm{CHO}$ cells $(400,000$ cells) was transferred from the cell culture flask to $1.5 \mathrm{~mL}$ centrifuge tubes. Each centrifuge tube was centrifuged for $5 \mathrm{~min}$ at $300 \times g$ and all supernatant was removed. An aliquot of $200 \mu \mathrm{L}$ of Gibco PBS (1×, calcium and magnesium-free, pH 7.2; Thermo Fisher Scientific) was added to the cell pellet and pipetted up and down five times to resuspend thoroughly. The centrifuge tube was centrifuged for $5 \mathrm{~min}$ at $300 \times \mathrm{g}$ and all supernatant was removed. The centrifuge tube was flicked to loosen the cell pellet from the bottom. An aliquot of $350 \mu \mathrm{L}$ of the buffer RLT (RNA lysis buffer; QIAGEN), which contains guanidine thiocyanate, was added to the pellet and pipetted up and down five times to mix thoroughly. The time it took to complete these steps was set to 15, 30, 45, or 60 min (Fig. 1). The entire cell suspension was immediately loaded onto a QIAshredder spin column (QIAGEN) and homogenized by centrifugation for 2 min in a microcentrifuge at maximum speed. The resultant homogenized lysate was kept at -80 ${ }^{\circ} \mathrm{C}$ until RNA isolation. Total RNA isolation was performed using a RNeasy mini kit (QIAGEN). The quality of total RNA was assessed by RIN values using the Agilent 2200 TapeStation (Agilent Technologies, Santa Clara, CA, USA). Libraries were prepared using a TruSeq Stranded mRNA Library Prep Kit (Illumina, San Diego, CA, USA). The finished cDNA libraries were quantified by using the Agilent 2200 TapeStation and sequenced on an Illumina NextSeq 500 platform with 75-bp single-end reads. The experiment procedures of this section were deposited to protocol.io with DOI: dx.doi.org/10.17504/protocols.io.57ng9me.

\section{Data analysis}

All the reads were deposited in the DDBJ Sequence Read Archive (accession number: DRA006016). The metadata associated with the data submission to DDBJ DRA006016 can be obtained from SRA Run Selector (https://www.ncbi.nlm.nih.gov/Traces/study/?acc=DRP004803\&o=acc_s\%3Aa). The quality of the raw reads was analyzed with FastQC (version 0.11.3; Andrews, 2010). All short reads were mapped to the CHO-K1 RefSeq assembly (22,516 sequences; RefSeq Assembly ID: GCF_000223135.1) and CHO-K1 mitochondrial DNA (1 sequence; RefSeq Assembly ID: GCF_000055695.1) using Bowtie2 (version 2.3.4.1; Langmead \& Salzberg, 2012) and quantified using RSEM (version 1.2.31, Li \& Dewey, 2011). Shannon's information entropy, which can be used to assess changes in transcriptome diversity (Ogata et al., 2012, 2015; Seekaki \& Ogata, 2017; Kannan et al., 2020; Liu et al., 2020) was calculated based on TPM (transcripts per million). Principal component analysis (PCA) based on TPM was performed with the prcomp 
158 function with scale = TRUE in the R Stats package (version 3.3.3; R Development Core Team,

159

160

161

162

163

164

165

166

167

168

169

170

171

172

173

174

175

176

177

178

179

180

181

182

183

184

185

186

187

188

189

190

191

192

193

194

195

196

197

2016). A hierarchical cluster analysis (HCA) dendrogram based on TPM using hclust function in the R Stats package (version 3.3.3, R Development Core Team, 2016). The hclust clustering was performed using distance metric based on the Spearman's rank correlation coefficient and clustering method "ward.D2". Differentially expressed gene (DEG) analyses were performed using the TCC package with edgeR on R console (Robinson and Oshlack, 2010; Sun et al., 2013). Genes with a false discovery rate (FDR) less than 0.05 were identified as differentially expressed. The identified DEGs were compared between $15 \mathrm{~min}$ and each other time period (30, 45, and $60 \mathrm{~min}$ ) on each cultivation day and the numbers of common DEGs among the four different days were counted. Orthology of CHO-K1 RefSeq genes and genes associated with apoptosis (249 genes with GO terms included "apoptotic" or "apoptosis", Supplemental Table S1) in the MGI (Mouse Genome Informatics, http://www.informatics.jax.org/) database was confirmed by using BLASTN searches. Then, the number of genes associated with apoptosis in the common DEGs was counted.

\section{Results}

To determine whether RNA-Seq data obtained from RNA samples that differ in the time lag from harvesting cells to protecting RNA (hereafter, "processing lag time") can be analyzed equally, we performed RNA-Seq of RNA isolated from CHO-S cells (at 3, 5, 6, and 7 days of cultivation) with RNA protected at various times $(15,30,45$, and $60 \mathrm{~min})$ following cell harvest, as shown in Fig. 1. High-quality total RNA was obtained from all samples (RIN values $>9.1$ ). We prepared cDNA libraries from total RNA and obtained 11 to 42 million sequence reads per sample. The mean base quality score in the Phred scale was 35.68 indicating that the base call accuracy was above $99.97 \%$. Sequence mapping rates to reference sequences ranged from $78.19 \%$ to $84.29 \%$.

Shannon's information entropy of RNA-Seq data of bach CHO-S cell culture was shown in Fig. 3. There were no significant differences in the value of Shannon's information entropy between samples from the same cultivation day with different processing lag times. On the other hands, the value of Shannon's information entropy decreased with cultivation. Decreasing Shannon's information entropy during the cultivation is also seen in other organisms used in industry (unpublished data).

PCA (Fig. 4) and HCA (Fig. 5) were conducted using RNA-Seq data mapped onto the CHO-K1 RefSeq assembly (GCF_000223135.1) and CHO-K1 mitochondrial DNA (GCF_000055695.1). PC1 (cumulative contribution ratio $(\%)=0.37$ ) appeared to be associated with cultivation day (Fig. 4A), but neither PC1 nor PC2 (cumulative contribution ratio $(\%)=0.10$ ) were associated with processing lag time (Fig. 4A-E). The HCA dendrogram showed that samples of the same cultivation day clustered together; there were four clusters associated with cultivation day (Fig.

PeerJ reviewing PDF | (2019:03:35644:1:0:NEW 15 Apr 2021) 
198

199

200

201

202

203

204

205

206

207

208

209

210

211

212

213

214

215

216

217

218

219

220

221

222

223

224

225

226

227

228

229

230

231

232

233

234

235

236

237

5). These results show that the gene expression was strongly associated with cultivation day, but the correlation between the gene expression and the processing lag time was not found. Even if processing lag times are different, global trends of gene expression were similar between samples from the same cultivation day.

Differentially expressed gene (DEG) analysis was conducted to explore the correlation between processing lag time $(15,30,45$, and $60 \mathrm{~min})$ and the gene expression. First, we compared the gene expression of RNA-Seq data between $15 \mathrm{~min}$ and each other time period (30, 45, and 60 min) on each cultivation day. The numbers of common DEGs among the four different days were 4 ( $15 \mathrm{~min}$ vs. $30 \mathrm{~min}$ ), 10 (15 $\mathrm{min}$ vs. $45 \mathrm{~min}$ ), and 23 (15 min vs. $60 \mathrm{~min}$ ) (Fig. 6). The number of common DEGs increased as the processing lag time increased (Fig. 6, Table 1). All 23 common DEGs, with the exception of LOC103159497, were upregulated in 30, 45, and 60 min samples compared with 15 min samples (Table 1, Supplemental Fig. S1). Twelve of the 23 common DEGs were orthologous to genes associated with apoptosis in the MGI database (Table 1).

PCA and HCA was then performed for genes identified as differentially expressed at least once in DEG analyses. In the PCA plot, PC1 and PC2 appeared to be associated with processing lag time of each cultivation day (Fig. 7A). PCA for each cultivation day also appeared to be associated with processing lag time (Fig. 7B-E). In the dendrogram (Fig. 8), we identified three major clusters associated with cultivation day: cluster 1 included all day 3 samples; cluster 2 included all day 5 samples; cluster 3 included all day 6 and 7 samples. It was consistent with the PCA (Fig. 7) that samples of days 6 and 7 were not clearly separated in the dendrogram. Then, each sub-cluster was associated with processing lag time. For example, cluster 3, which included day 6 and 7 samples, has three sub-clusters: cluster 3-1 included 15 min and 30 min samples; cluster 3-2 included $30 \mathrm{~min}$ and $45 \mathrm{~min}$ samples; cluster 3-3 included $45 \mathrm{~min}$ and $60 \mathrm{~min}$ samples. The influences of the processing lag time on the expression of transcriptome increased with cultivation day.

\section{Discussion}

To evaluate the influence of differences in processing lag time of cell samples (i.e., time until addition of RNA lysis buffer) on RNA-Seq, we examined gene expression changes when RNA lysis buffer was added at various times after the harvest of $\mathrm{CHO}$ cells. Regardless of the number of days of cell cultivation (3, 5, 6, and 7 days) or the processing lag time (15, 30, 45, and 60 $\mathrm{min}$ ), we obtained high-quality total RNA and high-quality sequence reads from all samples. The results of the value of Shannon's information entropy (Fig. 3), PCA (Fig. 4), and HCA (Fig. 5) showed that, for each cultivation day, the global trends of gene expression were similar between the samples with various processing lag times. In contrast, the expression levels of specific genes were affected by the processing lag time of the samples, despite the total RNA being of high 
238

239

240

241

242

243

244

245

246

247

248

249

250

251

252

253

254

255

256

257

258

259

260

261

262

263

264

265

266

267

268

269

270

271

272

273

274

275

276

277

quality. The number of common DEGs including apoptosis-associated genes increased as the processing lag time increased (Fig. 6, Table 1). In the dendrogram of the HCA performed for genes identified as differentially expressed at least once in DEG analyses (Fig. 8), each cluster consisted of several sub-clusters associated with processing lag times. Especially, cluster 3 including the samples of days 6 and 7 separated into three sub-clusters associated with processing lag times rather than cultivation day. Thus, we conclude that RNA lysis buffer should be added within 30 min after cell harvest. Otherwise, changes in the expression of some genes, especially genes associated with apoptosis, will occur.

Several studies have reported that the use of RNA samples with RIN values of around 5.0-7.0 does not negatively affect estimates of the gene expression profile (e.g., Fan et al., 2016; Shen et al., 2018) or de novo assembly (Kono et al., 2016). However, our results indicated that even if using high-quality RNA (RIN >9.1) for RNA-Seq, the gene expression levels of specific genes were significantly different between samples that share the same cultivation day but have different processing lag times. For instance, three genes of the AP-1 transcription factor complex (Fos, Jun, and Atf4) were detected as DEGs (Table 1), with higher expression as the processing lag time increased. AP-1 transcription factors regulate the expression of target genes involved in a wide range of cellular processes, such as proliferation, survival, and death. Fos is regarded as an early response gene to both biochemical and mechanical stress in several cell types (Peake et al., 2000; Kim et al., 2017). In the case of CHO cells, shear stress (Ranjan et al., 1996), virus infection (Zachos, Clements \& Conner, 1999), and cobalt chloride stimulation (Gong et al., 2001; Zou et al., 2001) have been known to induce AP-1 activation. Here, although the time spent on centrifuging and pipetting was equal for all samples (Fig. 1), the time placed in media or $1 \times$ PBS at room temperature was longer for samples with a longer processing lag time, suggesting that expression of AP-1 transcription factors increased in response to $1 \times \mathrm{PBS}$ stimulation and/or the conditions (temperature and $\mathrm{CO}_{2}$ concentration) outside the incubator.

Because the above results demonstrate that the processing lag time influences the expression level of specific genes, it is necessary to know not only the quality of the data but also how the experiment was performed when acquiring RNA-Seq data from SRA and investigating the expression level of a specific gene. However, detailed information on experimental operations, such as processing lag time, is usually not described in manuscripts.

In recent years, there has been growing focus on the importance of reporting detailed methods, resulting in several protocol repositories have been begun, such as Nature Protocol Exchange (https://protocolexchange.researchsquare.com/), Protocols.io (Teytelman et al., 2016), MethodsX (http://www.sciencedirect.com/science/journal/22150161), Journal of Visualized Experiments (JoVE, https:// www.jove.com/), and others. These repositories are an open-access, free platform for sharing, and discussing protocols and can be used for during research and manuscript submission to facilitate reproducibility. However, protocol repositories usually do not show the 
278 working time of the experiment, even if it is a time-sensitive experiment like RNA isolation.

279 Detailed protocols of this study including the processing time can be found at Protocols.io (see

280 Materials \& Methods).

281

282

283

284

285

286

287

288

289

290

291

292

293

294

295

296

297

298

299

300

301

302

303

304

305

306

307

308

309

310

311

312

313

314

315

316

317

Insufficient metadata will reduce the value of sequencing experiments by reducing the reproducibility of the study and its reuse for other analyses (Stevens et al., 2020). There are metadata standards for RNA-Seq such as the MINISEQE (Minimum Information about a highthroughput Nucleotide Sequencing Experiment), the ENCODE (Encyclopedia of DNA Elements) standards, or the International Human Epigenome Consortium (IHEC) metadata model. By following those metadata standards, or the checklist system for different types of data implemented by the European Nucleotide Archive (ENA), experimental details of RNA-Seq, such as the length of the RNA population being selected and whether rRNA populations were removed before library prep, can be described without missing. Furthermore, the BioSamples database at EMBL-EBI does not restrict the types of attributes used to describe sample metadata (Courtot et al., 2018), allowing submitters to choose attributes freely. However, those metadata standards for RNA-Seq do not have attributes corresponding to the working time of RNA isolation. The metadata associated with this study including the processing time of RNA samples described according to the ENCODE standards can be obtained from SRA Run Selector (see Material \& Methods).

Although efforts have been made regarding the description to improve the reproducibility of experiments, it is difficult to describe the time required for individual steps and the number of pipetting operations in protocols or metadata. In the future, it would be ideal to employ humanoid robots that can use pipettes, vortex mixers, incubators, refrigerators, and centrifuges for experiments. A robotic crowd (or cloud) biology laboratory (RCBL; Yachie, Robotic Biology Consortium \& Natsume, 2017) makes it possible to implement materials and methods complementary and robustly (Ochiai et al., 2020). The development of a humanoid robotic platform for sample processing would contribute to accurate recording and improve reproducibility of experiment operations.

\section{Conclusions}

In this study, we showed that differences in the time lag from harvesting cells to protecting RNA affect the expression level of specific genes in RNA-seq experiments. As discussed above, the data submitter needs to provide detailed information of the experiment together with the sequence reads. The database user needs to be able to investigate the validity of the data including potential problems with how the experiment was performed when acquiring HTS data from the database. Expression levels of the 23 DEGs identified in this study could potentially be used to help check whether the RNA of biological samples was properly treated. 


\section{Acknowledgements}

320

321

322

323

324

325

326

327

328

329

330

331

332

333

334

335

336

337

338

339

340

341

342

343

344

345

346

347

348

349

350

351

352

353

354

355

356

357

358

359

360

361

362

I am grateful to Dr. N. Ogata for his assistance with the experiments and useful discussions.

\section{References}

Alnasir J, Shanahan HP. 2015. Investigation into the annotation of protocol sequencing steps in the sequence read archive. Gigascience 4:23. DOI: 10.1186/s13742-015-0064-7.

Andrews S. 2010. FastQC: a quality control tool for high throughput sequence data. Available at https://www.bioinformatics.babraham.ac.uk/projects/fastqc (accessed 4 April 2018)

Carpenter AE, Jones TR, Lamprecht MR, Clarke C, Kang IH, Friman O, Guertin DA, Chang JH, Lindquist RA, Moffat J, Golland P, Sabatini DM. 2006. CellProfiler: image analysis software for identifying and quantifying cell phenotypes. Genome Biology 7:R100. DOI: 10.1186/gb-2006-7-10-r100.

Courtot M, Cherubin L, Faulconbridge A, Vaughan D, Green M, Richardson D, Harrison P, Whetzel PL, Parkinson H, Burdett T. 2018. BioSamples database: an updated sample metadata hub. Nucleic Acids Research 47:D1172-D1178. DOI: 10.1093/nar/gky1061.

Fan J, Khanin R, Sakamoto H, Zhong Y, Michael C, Pena D, Javier B, Wood LD, IacobuzioDonahue CA. 2016. Quantification of nucleic acid quality in postmortem tissues from a cancer research autopsy program. Oncotarget 7:66906-66921. DOI: 10.18632/oncotarget.11836.

Gong P, Hu B, Stewart D, Ellerbe M, Figueroa YG, Blank V, Beckman BS, Alam J. 2001. Cobalt induces heme oxygenase-1 expression by a hypoxia-inducible factor-independent mechanism in Chinese hamster ovary cells: regulation by Nrf2 and MafG transcription factors. Journal of Biological Chemistry 276:27018-27025. DOI: 10.1074/jbc.M103658200.

Kannan S, Farid M, Lin BL, Miyamoto M, Kwon C. 2020. Transcriptomic entropy benchmarks stem cell-derived cardiomyocyte maturation against endogenous tissue at single cell level. Cold Spring Harbor Laboratory:2020.04.02.022632. DOI: 10.1101/2020.04.02.022632.

Kim J, Montagne K, Nemoto H, Ushida T, Furukawa KS. 2017. Hypergravity down-regulates cfos gene expression via ROCK/Rho-GTP and the PI3K signaling pathway in murine ATDC5 chondroprogenitor cells. PloS One 12:e0185394. DOI: 10.1371/journal.pone.0185394.

Kircher M, Kelso J. 2010. High-throughput DNA sequencing--concepts and limitations. Bioessays 32:524-536. DOI: 10.1002/bies.200900181

Kirkconnell KS, Paulsen MT, Magnuson B, Bedi K, Ljungman M. 2016. Capturing the dynamic nascent transcriptome during acute cellular responses: The serum response. Biology Open 5:837-847. DOI: 10.1242/bio.019323

Kodama Y, Shumway M, Leinonen R, International Nucleotide Sequence Database Collaboration. 2012. The Sequence Read Archive: explosive growth of sequencing data. Nucleic Acids Research 40:D54-D56. DOI: 10.1093/nar/gkr854.

Kono N, Nakamura H, Ito Y, Tomita M, Arakawa K. 2016. Evaluation of the impact of RNA preservation methods of spiders for de novo transcriptome assembly. Molecular Ecology Resources 16:662-672. DOI: 10.1111/1755-0998.12485. 
363

364

365

366

367

368

369

370

371

372

373

374

375

376

377

378

379

380

381

382

383

384

385

386

387

388

389

390

391

392

393

394

395

396

397

398

399

400

401

402

403

404

405

406

407

408

Leinonen R, Sugawara H, Shumway M. 2010. The Sequence Read Archive. Nucleic Acids Research 39:D19-D21. DOI: 10.1093/nar/gkq1019.

Langmead B, Salzberg SL. 2012. Fast gapped-read alignment with Bowtie 2. Nature Methods 9:357-359. DOI: 10.1038/nmeth.1923.

Li B, Dewey CN. 2011. RSEM: accurate transcript quantification from RNA-Seq data with or without a reference genome. BMC Bioinformatics 12:323. DOI: 10.1186/1471-2105-12323.

Liu B, Li C, Li Z, Wang D, Ren X, Zhang Z. 2020. An entropy-based metric for assessing the purity of single cell populations. Nature communications 11:3155. DOI: $10.1038 / \mathrm{s} 41467-$ 020-16904-3.

Losada L, Barker BM, Pakala S, Pakala S, Joardar V, Zafar N, Mounaud S, Fedorova N, Nierman WC, Cramer RA. 2014. Large-scale transcriptional response to Hhypoxia in Aspergillus fumigatus observed using RNAseq identifies a novel hypoxia regulated ncRNA. Mycopathologia 178:331-339. DOI: 10.1007/s11046-014-9779-8.

Nakazato T, Ohta T, Bono H. 2013. Experimental design-based functional mining and characterization of high-throughput sequencing data in the sequence read archive. PloS One 8:e77910. DOI: 10.1371/journal.pone.0077910.

Ochiai K, Motozawa N, Terada M, Horinouchi T, Masuda T, Kudo T, Kamei M, Tsujikawa A, Matsukuma K, Natsume T, Kanda GN, Takahashi M, Takahashi K. 2020. A Variable Scheduling Maintenance Culture Platform for Mammalian Cells. SLAS technology:2472630320972109. DOI: 10.1177/2472630320972109.

Ogata N, Yokoyama T, Iwabuchi K. 2012. Transcriptome Responses of Insect Fat Body Cells to Tissue Culture Environment. PloS one 7:e34940. DOI: 10.1371/journal.pone.0034940.

Ogata N, Kozaki T, Yokoyama T, Hata T, Iwabuchi K. 2015. Comparison between the Amount of Environmental Change and the Amount of Transcriptome Change. PloS one 10:e0144822. DOI: 10.1371/journal.pone.0144822.

Ohta T, Nakazato T, Bono H. 2017. Calculating the quality of public high-throughput sequencing data to obtain a suitable subset for reanalysis from the Sequence Read Archive. Gigascience 6:1-8. DOI: 10.1093/gigascience/gix029.

Peake MA, Cooling LM, Magnay JL, Thomas PB, El Haj AJ. 2000. Selected contribution: regulatory pathways involved in mechanical induction of c-fos gene expression in bone cells. Journal of applied physiology 89:2498-2507. DOI: 10.1152/jappl.2000.89.6.2498.

R Development Core Team. 2016. R: A language and environment for statistical computing. Vienna: R Foundation for Statistical Computing.

Ranjan V, Waterbury R, Xiao Z, Diamond SL. 1996. Fluid shear stress induction of the transcriptional activator c-fos in human and bovine endothelial cells, HeLa, and Chinese hamster ovary cells. Biotechnology and Bioengineering 49:383-390. DOI: 10.1002/(SICI)1097-0290(19960220)49:4<383::AID-BIT4>3.0.CO;2-L.

Robinson MD, Oshlack A. 2010. A scaling normalization method for differential expression analysis of RNA-seq data. Genome Biology 11:R25. DOI: 10.1186/gb-2010-11-3-r25.

Seekaki P, Ogata N. 2017. Calculating Kolmogorov Complexity from the Transcriptome Data. In: Intelligent Computing Theories and Application. Springer International Publishing, 529-540. DOI: 10.1007/978-3-319-63312-1_46.

Shen Y, Li R, Tian F, Chen Z, Lu N, Bai Y, Ge Q, Lu Z. 2018. Impact of RNA integrity and blood sample storage conditions on the gene expression analysis. OncoTargets and Therapy 11:3573-3581. DOI: 10.2147/OTT.S158868.

PeerJ reviewing PDF | (2019:03:35644:1:0:NEW 15 Apr 2021) 
409 Stevens I, Mukarram AK, Hörtenhuber M, Meehan TF, Rung J, Daub CO. 2020. Ten simple

410

411

412

413

414

415

416

417

418

419

420

421

422

423

424

425

426 rules for annotating sequencing experiments. PLoS computational biology 16:e1008260. DOI: 10.1371/journal.pcbi.1008260.

Sun J, Nishiyama T, Shimizu K, Kadota K. 2013. TCC: an R package for comparing tag count data with robust normalization strategies. BMC Bioinformatics 14:219. DOI: 10.1186/1471-2105-14-219.

Teytelman L, Stoliartchouk A, Kindler L, Hurwitz BL. 2016. Protocols.io: virtual communities for protocol development and discussion. PloS Biology 14:e1002538. DOI: 10.1371/journal.pbio.1002538.

Yachie N, Robotic Biology Consortium, Natsume T. 2017. Robotic crowd biology with Maholo LabDroids. Nature Biotechnology 35:310-312. DOI: 10.1038/nbt.3758.

Zachos G, Clements B, Conner J. 1999. Herpes simplex virus type 1 infection stimulates p38/cJun N-terminal mitogen-activated protein kinase pathways and activates transcription factor AP-1. Journal of Biological Chemistry 274:5097-5103. DOI: 10.1074/jbc.274.8.5097.

Zou W, Yan M, Xu W, Huo H, Sun L, Zheng Z, Liu X. 2001. Cobalt chloride induces PC12 cells apoptosis through reactive oxygen species and accompanied by AP-1 activation. Journal of Neuroscience Research 64:646-653 DOI: 10.1002/jnr.1118. 


\section{Table 1 (on next page)}

Common differentially expressed genes (DEGs) among four different days 


\begin{tabular}{|c|c|c|c|c|c|c|}
\hline \multirow[t]{2}{*}{ Gene name } & \multirow[t]{2}{*}{ CHO-Refseq } & \multirow[t]{2}{*}{ MGI ID } & \multirow[t]{2}{*}{ GO terms included "apoptosis" or "apoptotic" } & \multicolumn{3}{|c|}{ Common DEGs among days } \\
\hline & & & & $\begin{array}{l}15 \min \text { vs. } 30 \\
\min \end{array}$ & $\begin{array}{l}15 \min \text { vs. } 45 \\
\min \end{array}$ & $15 \mathrm{~min}$ vs. $60 \mathrm{~min}$ \\
\hline Atf4 & XM_007654285.1, NM_001246812.1 & MGI:88096 & GO:0043525, GO:0070059, GO:1905461 & - & - & + \\
\hline Btg2 & XM_003505756.2 & MGI:108384 & GO:0043066, GO:0043524 & - & - & + \\
\hline Chub2 & NM_001244378.1 & $*$ & - & - & + & + \\
\hline Cyr61 & XM_007653329.1, XM_003512942.2 & MGI:88613 & $\begin{array}{lll}\text { GO:0003278, } & \text { GO:0043065, } & \text { GO:0043066, } \\
\text { GO:0043280 } & & \end{array}$ & - & - & + \\
\hline Ddx 5 & XM_003501860.2 & MGI:105037 & GO:0072332 & - & - & + \\
\hline Dusp5 & XM_007653051.1 & $*$ & - & - & - & + \\
\hline Egr1 & XM_003502541.2 & MGI:95295 & GO:0042981, GO:0043525 & + & + & + \\
\hline Egr2 & XM_003515916.2 & * & - & - & + & + \\
\hline Fos & NM_001246683.1 & $*$ & - & + & + & + \\
\hline Fosb & XM_007645972.1 & $*$ & - & - & - & + \\
\hline H3f3b & XM_003506831.2 & $*$ & - & - & - & + \\
\hline Ier3 & XM_003505887.2 & MGI:104814 & GO:0008630, GO:0043066, GO:1901029 & - & + & + \\
\hline Ier5 & XM_007640860.1 & $*$ & - & - & - & + \\
\hline Jun & XM_007643818.1 & MGI:96646 & $\begin{array}{lll}\text { GO:0043065, } & \text { GO:0043066, } & \text { GO:0043524, } \\
\text { GO:0043525 } & & \end{array}$ & - & + & + \\
\hline Junb & XM_007642561.1 & $*$ & - & + & + & + \\
\hline Klf6 & XM_003494905.2, XM_003515975.2 & $*$ & - & - & - & + \\
\hline $\begin{array}{l}\text { LOC1031594 } \\
97\end{array}$ & XR_484059.1 & * & - & + & + & + \\
\hline Plk2 & XM_003496201.2 & MGI:1099790 & GO:0043066, GO:0071866 & - & - & + \\
\hline Ppp1r15a & XM_007649270.1 & MGI:1927072 & GO:0006915 & - & - & + \\
\hline Sgk1 & XM_003511162.2 & MGI:1340062 & GO:0006915, GO:0043066 & - & - & + \\
\hline Srsf5 & $\begin{array}{l}\text { XM_003498255.2, } \\
\text { XR_480198.1 }\end{array}$ & * & - & - & + & + \\
\hline
\end{tabular}




\section{PeerJ}

\begin{tabular}{|c|c|c|c|c|c|c|c|c|}
\hline Vegfa & XM_007642338.1 & MGI:103178 & $\begin{array}{l}\text { GO:0043066, } \\
\text { GO:2001237 }\end{array}$ & GO:0043154, & GO:0043524, & - & - & + \\
\hline Zfp36 & XM_007644391.1 & MGI:99180 & GO:1902172 & & & - & + & + \\
\hline
\end{tabular}

2 An asterisk symbol $(*)$ indicates that the gene was not included in the MGI (Mouse Genome Informatics) database.

3 A plus symbol (+) indicates that the gene was detected as a common DEG.

4 A minus symbol (-) indicates that the gene was not detected as a common DEG. 


\section{Figure 1}

Time course illustration of the experiment.

RNA in CHO cells was protected at different times (15, 30, 45, and $60 \mathrm{~min}$ ) after cell harvest by addition of RNA lysis buffer (buffer RLT, QIAGEN). Except for the 15 min samples, cells were left in media or 1X PBS at room temperature for 4-13 min or 11-33 min, respectively.

Cells suspended in media or $1 \times$ PBS were centrifuged at $300 \times \mathrm{g}$. Cells were stored in media at room temperature.

The cell pellet was resuspended gently with $200 \mu \mathrm{L}$ of 1 xPBS.

Cells were stored in $1 \times$ PBS at room temperature.

$15 \min \stackrel{0}{ }{ }^{2} \quad 10 \quad 15$

All supernatant was removed and the centrifuge tube was flicked to loosen the pellet from the bottom. Then, $350 \mu \mathrm{L}$ buffer RLT was added.

$\begin{array}{lllllll}0 & 5 & 10 & 15 & 20 & 25 & 30\end{array}$

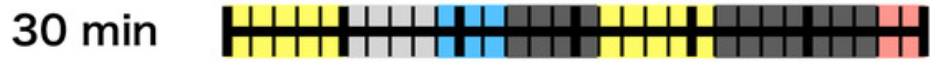

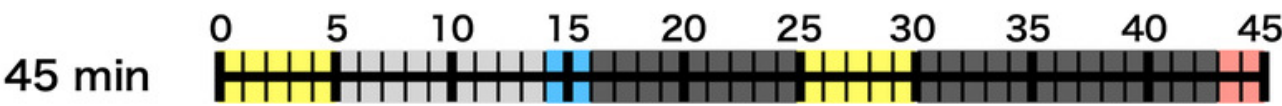

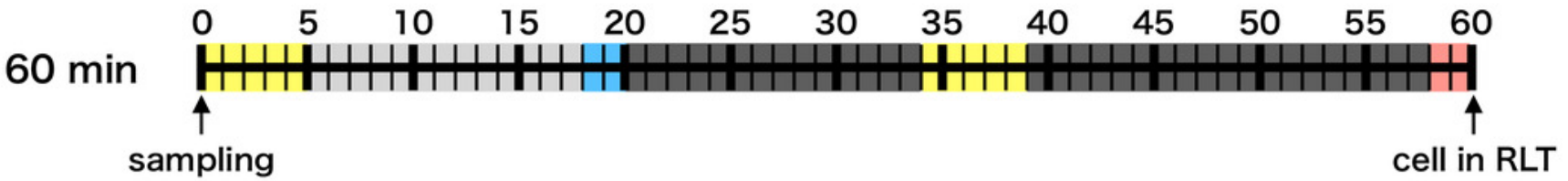


Figure 2

Growth curve of CHO-S cells.

Cells used for the experiment are indicated by filled squares.

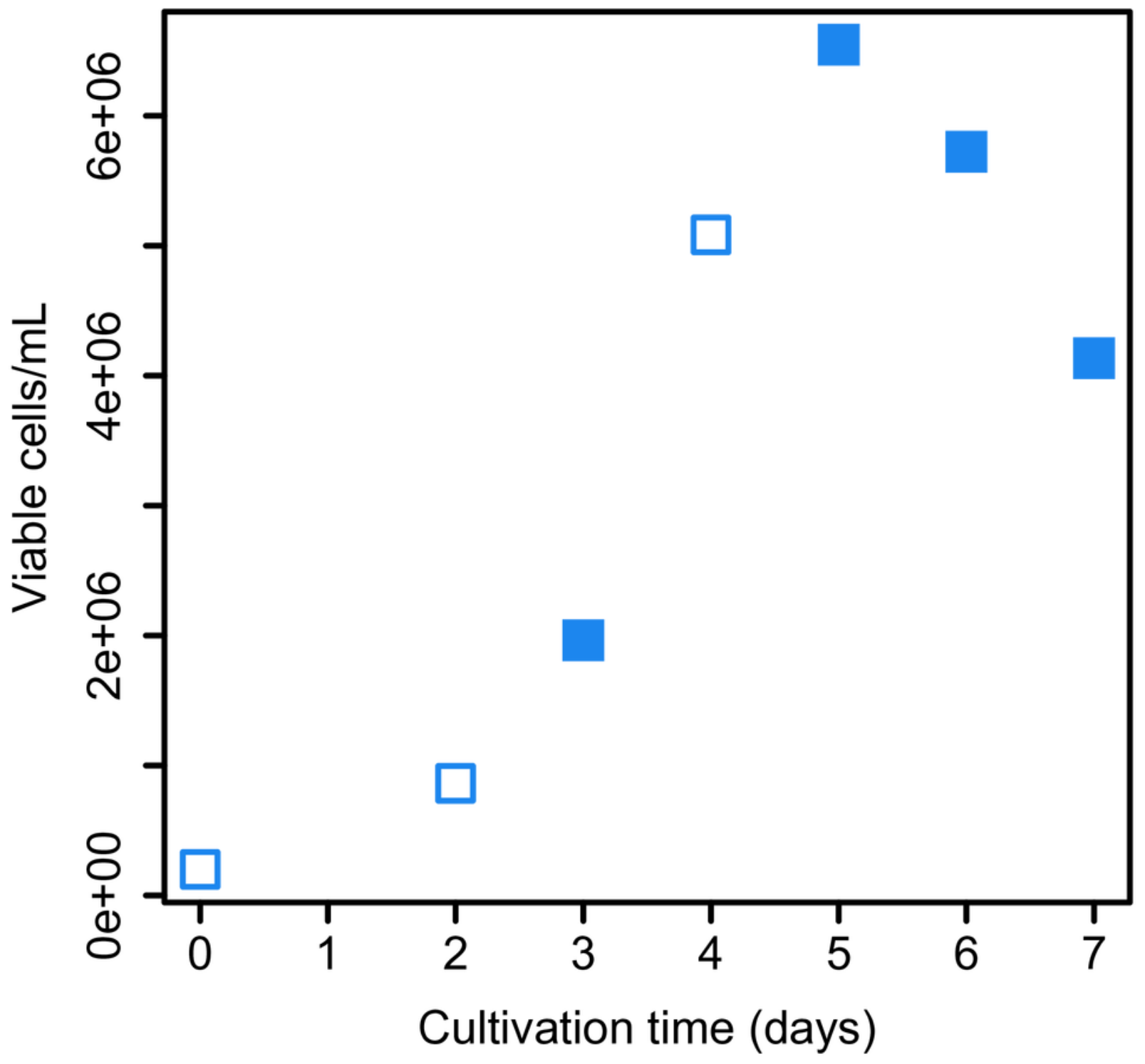


Figure 3

Scatter plot of cultivation dayvs. Shannon's information entropy.

There were no significant differences in the value of Shannon's information entropy between samples from the same cultivation day with different processing lag times. 


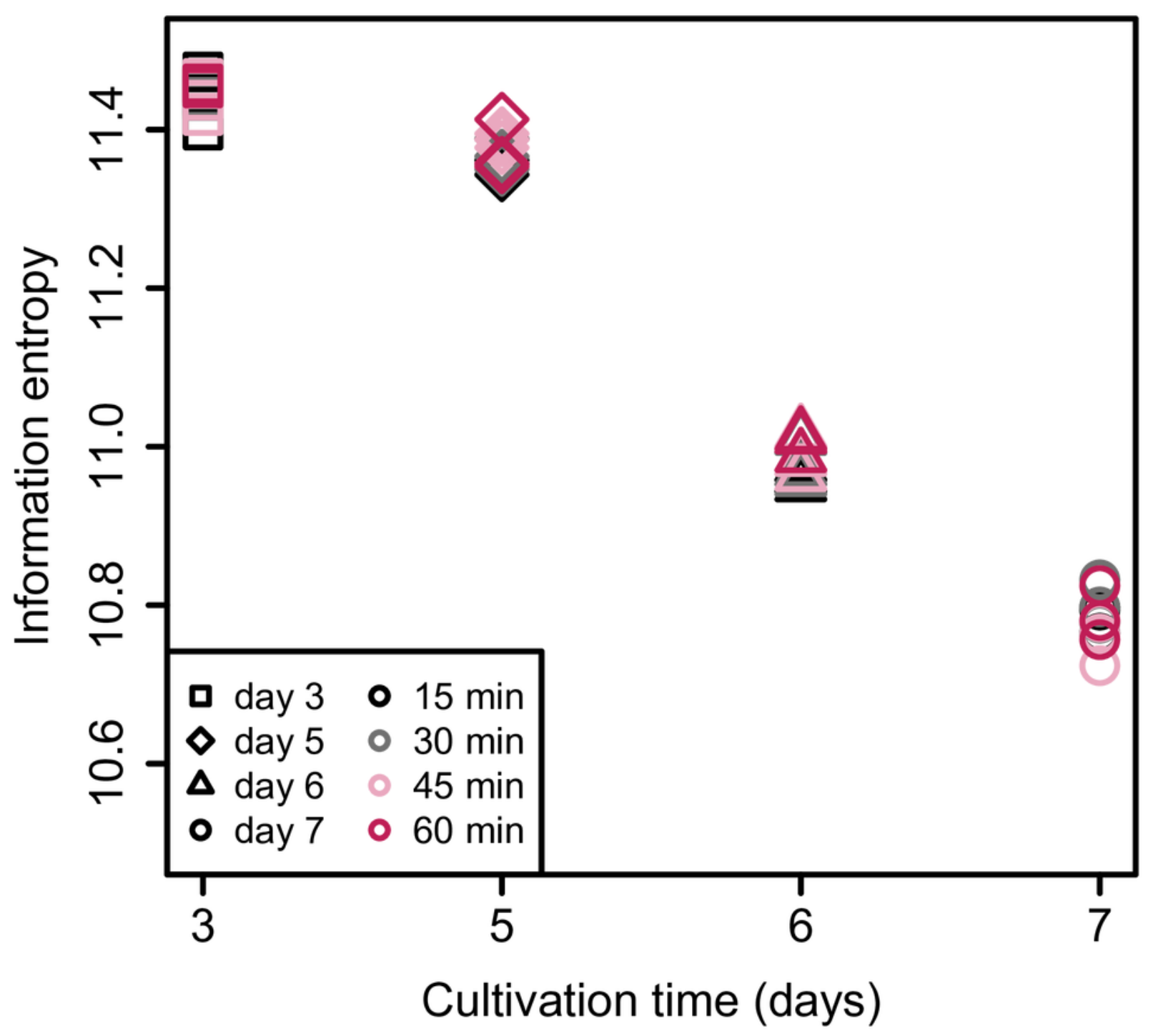


Figure 4

Score plot of principal component analysis (PCA) using PC1 and PC2.

PCA plot of the 48 samples included in the study based on data from CHO-K1 RefSeq assembly (GCF_000223135.1) and CHO-K1 mitochondrial DNA (GCF_000055695.1) with at least one mapped read on at least one of each sample. PC1 appeared to be associated with cultivation day, but neither PC1 nor PC2 were associated with processing lag time. (A) All 48 samples. Different colors identify different processing lag times, while different shapes identify different cultivation day. (B), (C), (D), and (E) Samples from day 3, 5, 6, and 7, respectively. Legend is same as $(A)$.
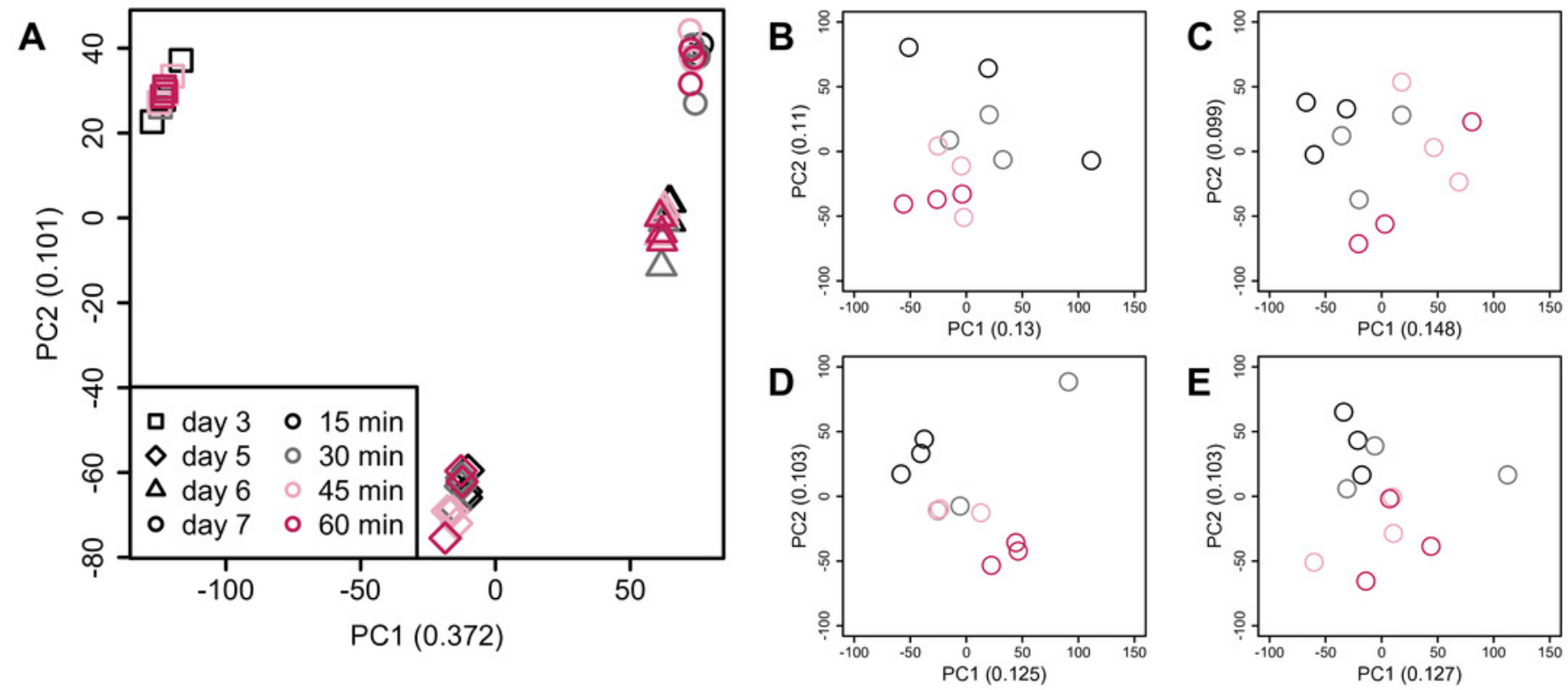


\section{Figure 5}

Dendrogram resulting from hierarchical clustering analysis (HCA).

HCA was performed based on data from CHO-K1 RefSeq assembly (GCF_000223135.1) and CHO-K1 mitochondrial DNA (GCF_000055695.1) with at least one mapped read on at least one of each sample. Each sample is indicated by the number of days of cultivation, the number of minutes of processing lag-time, and the technical replicate number. The dendrogram showed that samples of the same cultivation day clustered together; there were four clusters associated with cultivation day.

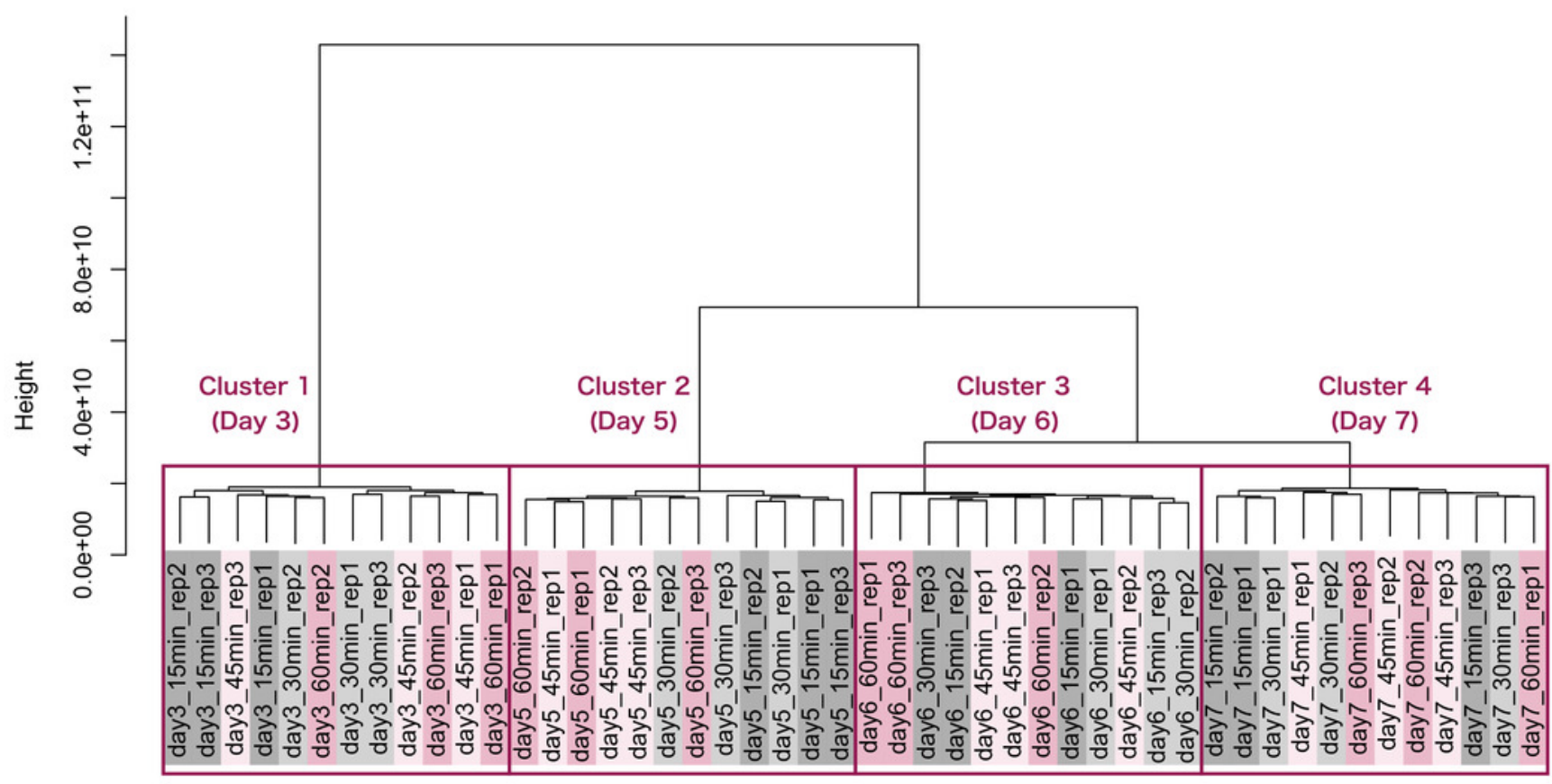


Figure 6

Venn diagrams of differentially expressed genes (DEGs).

The numbers of genes that were DEGs between different processing lag times among all four different days were (A) 4 (15 min vs. $30 \mathrm{~min}$ ), (B) 10 (15 min vs. $45 \mathrm{~min}$ ), and (C) 23 (15 min vs. $60 \mathrm{~min}$ ). (D) 4 common DEGs were detected among (a) $15 \mathrm{~min}$ vs. $30 \mathrm{~min}$, (b) $15 \mathrm{~min}$ vs. $45 \mathrm{~min}$, and (c) 15 min vs. $60 \mathrm{~min}$. 
A

B

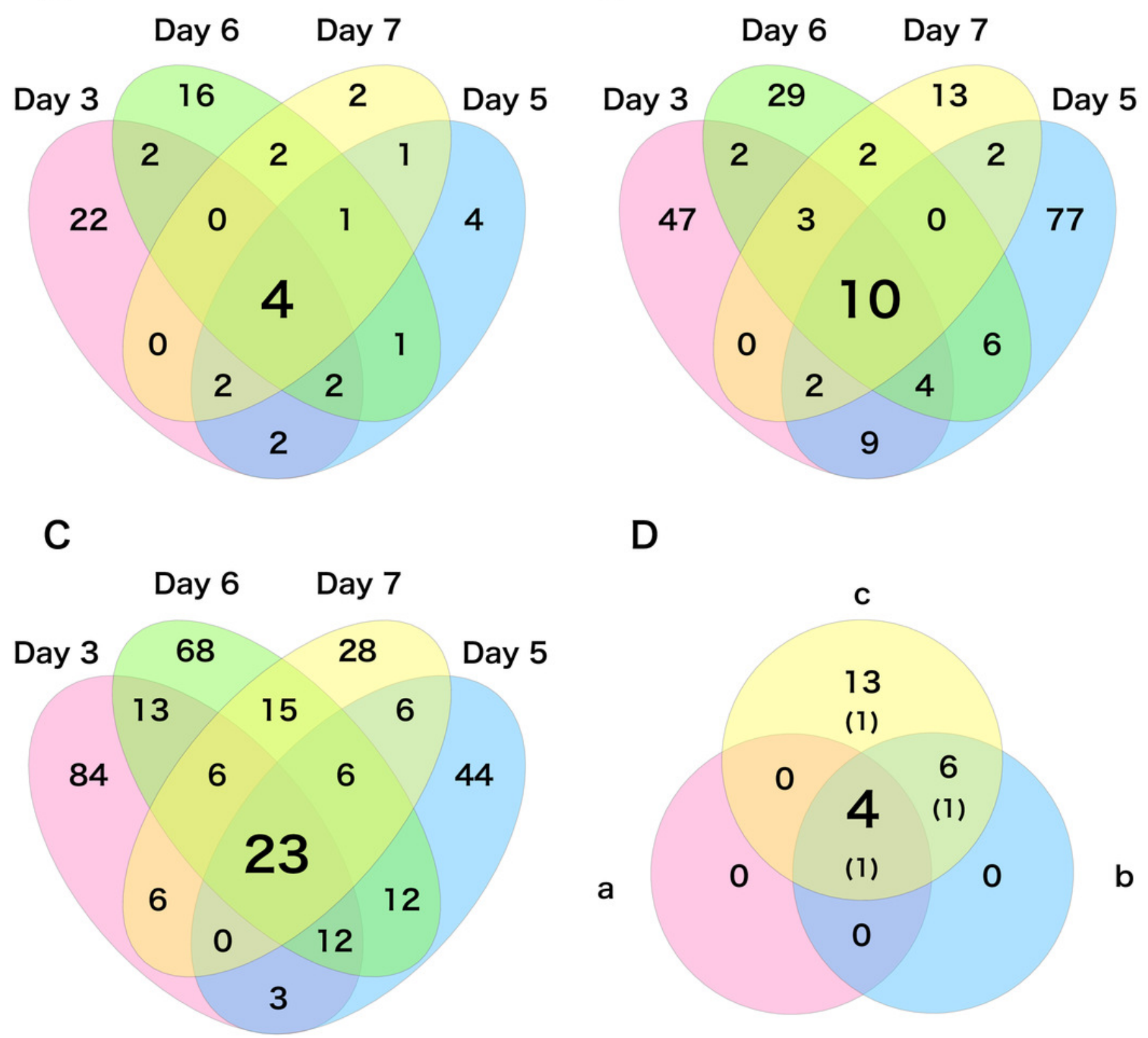


Figure 7

Score plot of principal component analysis (PCA) using PC1 and PC2.

PCA plot of the 48 samples included in the study based on data from genes identified as differentially expressed at least once in DEG analyses. PC1 and PC2 appeared to be associated with processing lag time of each cultivation day. (A) All 48 samples. (B), (C), (D), and (E) Samples from day 3, 5, 6, and 7, respectively. Legend is same as Fig. 4.
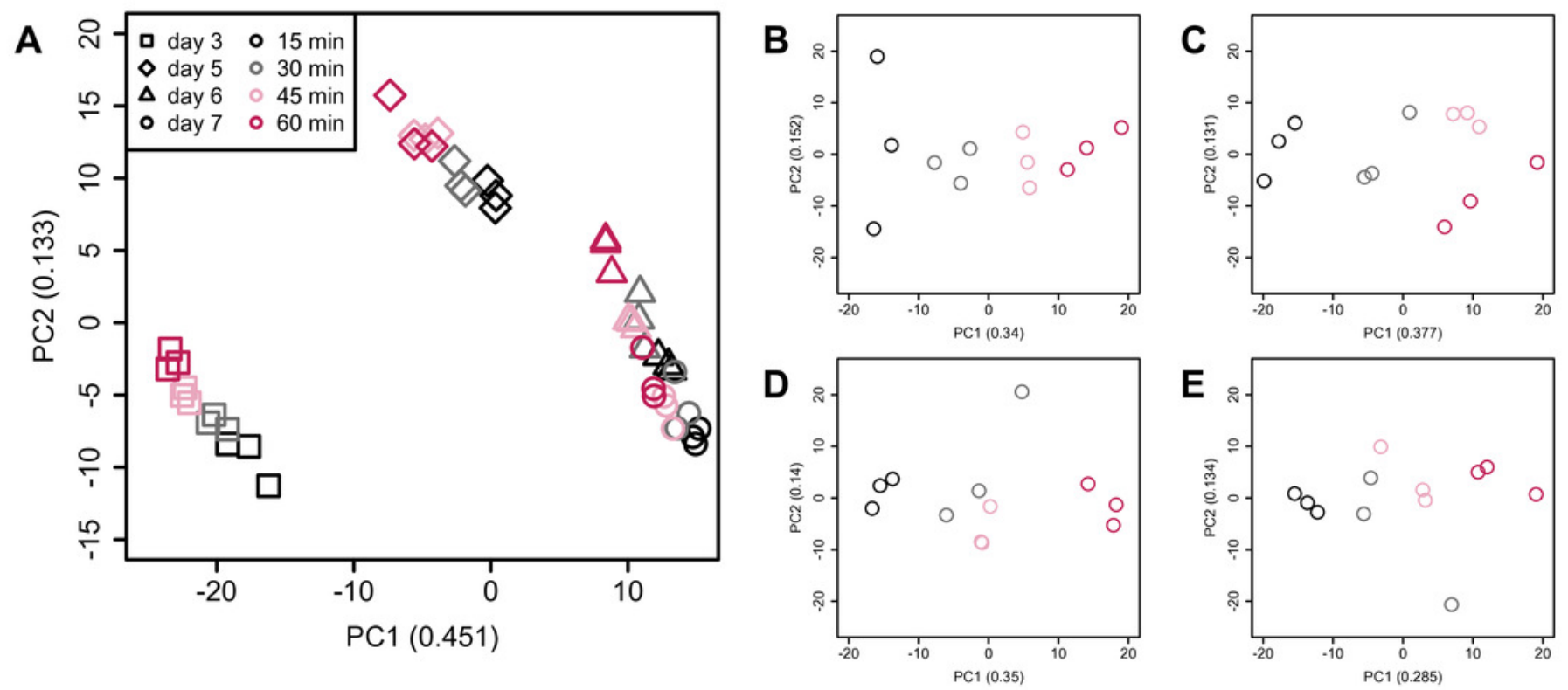


\section{Figure 8}

Dendrogram resulting from hierarchical clustering analysis (HCA).

HCA was performed based on data from genes identified as differentially expressed at least once in DEG analyses. Each sample is indicated by the number of days of cultivation, the number of minutes of processing lag-time, and the technical replicate number. The sample names are as described in the Fig. 5 legend. There are three major clusters associated with cultivation day and seven sub-clusters associated with processing lag time.

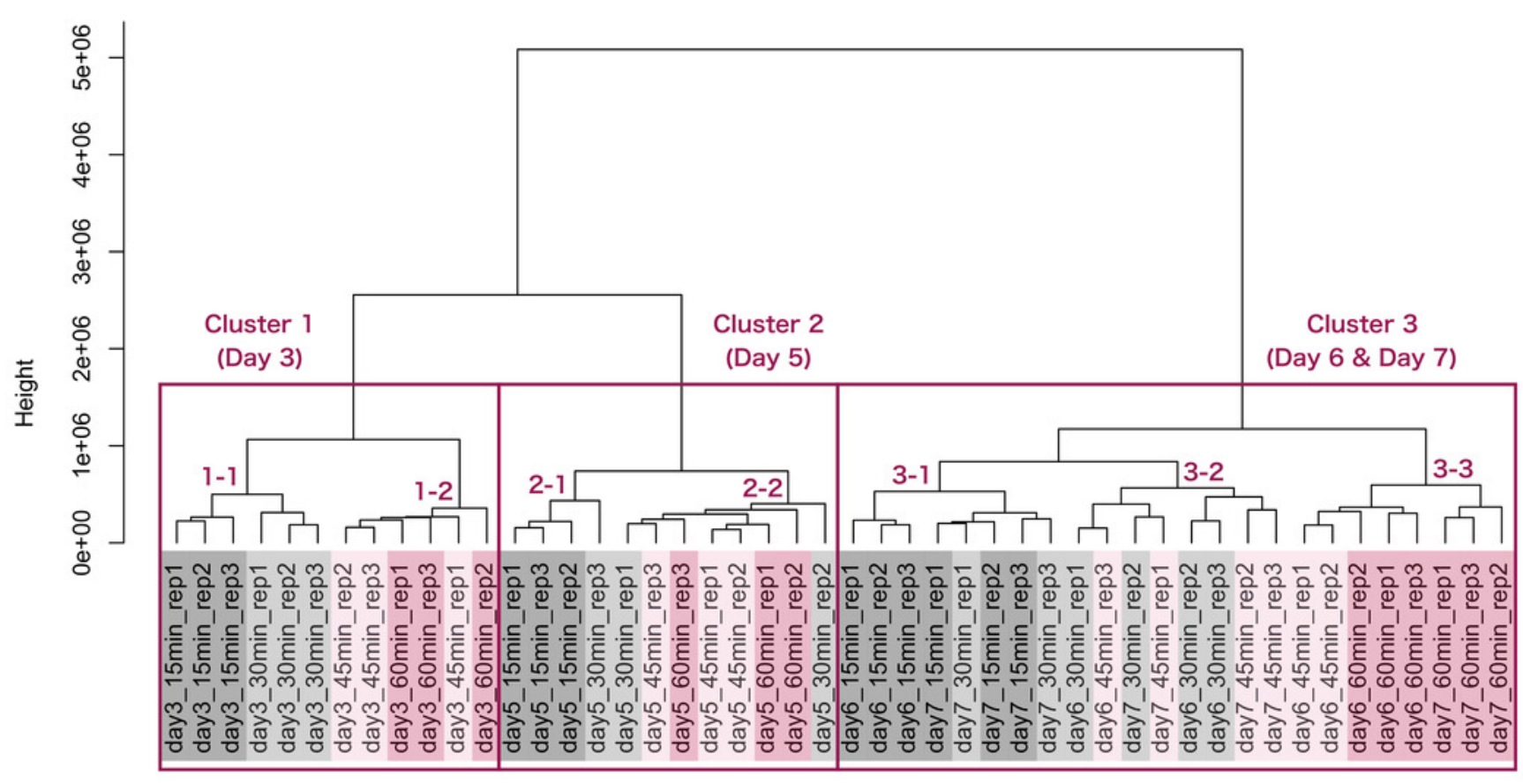

